\title{
THE FIRST SUBSTELLAR SUBDWARF? DISCOVERY OF A METAL-POOR L DWARF WITH HALO KINEMATICS
}

\author{
Adam J. Burgasser, ${ }^{1,2}$ J. Davy Kirkpatrick, ${ }^{3}$ Adam Burrows,${ }^{4}$ James Liebert, ${ }^{4}$ I. Neill Reid, ${ }^{5}$ \\ John E. Gizis, ${ }^{6}$ Mark R. McGovern, ${ }^{1}$ L. Prato, ${ }^{1}$ and Ian S. McLean ${ }^{1}$ \\ Received 2003 March 5; accepted 2003 April 9
}

\begin{abstract}
We present the discovery of the first L-type subdwarf, 2MASS J05325346+8246465. This object exhibits enhanced collision-induced $\mathrm{H}_{2}$ absorption, resulting in blue near-infrared (NIR) colors $\left(J-K_{s}=0.26 \pm\right.$ 0.16). In addition, strong hydride bands in the red optical and NIR, weak TiO absorption, and an optical/ $J$-band spectral morphology similar to the L7 DENIS 0205-1159AB imply a cool, metal-deficient atmosphere. We find that 2MASS $0532+8246$ has both a high proper motion, $\mu=2$ ". $60 \pm 0$ "' $15 \mathrm{yr}^{-1}$, and a substantial radial velocity, $v_{\mathrm{rad}}=-195 \pm 11 \mathrm{~km} \mathrm{~s}^{-1}$, and its probable proximity to the $\operatorname{Sun}(d=10-30 \mathrm{pc})$ is consistent with halo membership. Comparison to subsolar-metallicity evolutionary models strongly suggests that 2MASS $0532+8246$ is substellar, with a mass of $0.077 \lessgtr M \lessgtr 0.085 M_{\odot}$ for ages $10-15$ Gyr and metallicities $Z=0.1-0.01 Z_{\odot}$. The discovery of this object clearly indicates that star formation occurred below the hydrogen burning mass limit at early times, consistent with prior results indicating a flat or slightly rising mass function for the lowest mass stellar subdwarfs. Furthermore, 2MASS $0532+8246$ serves as a prototype for a new spectral class of subdwarfs, additional examples of which could be found in NIR proper-motion surveys.
\end{abstract}

Subject headings: infrared: stars — solar neighborhood — stars: chemically peculiar stars: individual (2MASS J05325346+8246465) — stars: low-mass, brown dwarfs — subdwarfs

On-line material: color figure

\section{INTRODUCTION}

Subdwarfs are metal-deficient stars, classically defined as lying below the stellar main sequence in optical colormagnitude diagrams (Kuiper 1939). These objects are in fact not subluminous but rather hotter (i.e., bluer in optical colors) than equivalent-mass main-sequence dwarfs, a consequence of their reduced metal opacity (Chamberlin \& Aller 1951; Sandage \& Eggen 1959). Cool subdwarfs (spectral types $\mathrm{sdK}$ and $\mathrm{sdM})$ are typically found to have halo kinematics $\left(\langle V\rangle=-202 \mathrm{~km} \mathrm{~s}^{-1}\right.$; Gizis 1997), and these objects are presumably relics of the early Galaxy, with ages of 10 15 Gyr. Because low-mass subdwarfs have lifetimes far in excess of the age of the Galaxy, they are important tracers of Galactic chemical history and are representatives of the first generations of star formation.

All of the coolest subdwarfs $([\mathrm{Fe} / \mathrm{H}] \sim-1.2 \pm 0.3)$ and extreme subdwarfs $([\mathrm{Fe} / \mathrm{H}] \sim-2.0 \pm 0.5$; Gizis 1997) currently known have been identified in optical proper-motion surveys, most notably Luyten's Half-Second Catalog (LHS; Luyten 1979a) and Two-Tenths Catalog (Luyten 1979b),

\footnotetext{
${ }^{1}$ Department of Physics and Astronomy, University of California, Los Angeles, CA 90095-1562; adam@astro.ucla.edu, mcgovern@astro.ucla.edu,lprato@astro.ucla.edu, mclean@astro.ucla.edu.

${ }^{2}$ Hubble Fellow.

${ }^{3}$ Infrared Processing and Analysis Center, MS 100-22, California Institute of Technology, Pasadena, CA 91125; davy@ipac.caltech.edu.

${ }^{4}$ Steward Observatory, University of Arizona, Tucson, AZ 85721; burrows@as.arizona.edu, liebert@as.arizona.edu.

${ }^{5}$ Space Telescope Science Institute, 3700 San Martin Drive, Baltimore, MD 21218;inr@stsci.edu.

${ }^{6}$ Department of Physics and Astronomy, University of Delaware, Newark, DE 19716; gizis@udel.edu.
}

the Automatic Plate Measuring Proper Motion Survey (Scholtz et al. 2000), and the Galactic plane survey of Lepine, Shara, \& Rich (2002). The substantial space velocities of halo subdwarfs allow them to stand out in these surveys among the overwhelming multitude of similarly faint but slowly moving background stars. However, only a handful of very cool subdwarfs with spectral types sdM6/ esdM6 and later ${ }^{7}$ have been found (Gizis 1997; Gizis \& Reid 1997; Schweitzer et al. 1999; Lepine, Rich, \& Shara 2003; Lepine, Shara, \& Rich 2003). These stars exhibit characteristic spectral signatures of strong metal hydrides $(\mathrm{CaH}$, $\mathrm{MgH}, \mathrm{AlH}$, and $\mathrm{FeH}$ ), weak and/or absent metal oxides (TiO, VO, and CO), and enhanced collision-induced $\mathrm{H}_{2}$ absorption (CIA: Mould \& Hyland 1976; Liebert \& Probst 1987; Saumon et al. 1994; Leggett et al. 2000). With $T_{\text {eff }} \gtrsim 2900 \mathrm{~K}$ (Leggett et al. 2000), the coolest subdwarfs known have masses just above the hydrogen burning minimum mass (HBMM) ${ }^{8}$ which ranges from $0.072 M_{\odot}$ for solar composition $\left(Z=Z_{\odot}\right)$ to $0.092 M_{\odot}$ for $Z=0$ (Chabrier \& Baraffe 1997; Burrows et al. 2001).

In contrast, hundreds of significantly cooler Population I, or disk, dwarfs have been identified, ${ }^{9}$ predominately in the wide-field optical and near-infrared (NIR) surveys Deep Near Infrared Survey of the Southern Sky (DENIS; Epchtein et al. 1997), the Two Micron All Sky Survey (2MASS; Skrutskie et al. 1997), and the Sloan Digital Sky

\footnotetext{
${ }^{7}$ Based on the Gizis (1997) classification scheme.

${ }^{8}$ The HBMM is defined here as the minimum mass for which core hydrogen fusion balances luminosity at ages later than $10 \mathrm{Gyr}$.

${ }^{9} \mathrm{~A}$ listing of known $\mathrm{M}, \mathrm{L}$, and $\mathrm{T}$ dwarfs is maintained by $\mathrm{J}$. D. Kirkpatrick at http://spider.ipac.caltech.edu/staff/davy/ARCHIVE/ index.html.
} 
Survey (SDSS; York et al. 2000). These discoveries include many dozens of substellar objects and extend well beyond the M spectral class into the L (Kirkpatrick et al. 1999; Martín et al. 1999) and the T (Burgasser et al. 2002; Geballe et al. 2002) classes. The success of recent surveys in identifying very cool dwarf star and brown dwarfs derives from their use of red-sensitive CCDs and infrared array detectors. As we proceed to cooler temperatures, the majority of emitted flux from both disk dwarfs and subdwarfs moves to NIR wavelengths. Unfortunately, proper-motion surveys of sufficient temporal breadth to identify halo stars rely primarily on optical, typically photographic imaging. Factoring in their intrinsic rarity $(0.3 \%$ number density compared with disk dwarfs; Reid \& Hawley 2000) and substantial evolution toward lower luminosities (Burrows et al. 1993; Chabrier \& Baraffe 1997), it is clear why few very cool subdwarfs have been found.

In this article, we present the discovery of a new cool subdwarf, identified in the 2MASS database. This object, 2MASS J05325346+8246465, ${ }^{10}$ is a high-motion source with a spectral morphology consistent with a metal deficient L dwarf. In $\S 2$ we discuss the identification of 2 MASS $0532+8246$ and subsequent NIR and optical observations obtained at the Palomar 60 inch Telescope. In $\S 3$ we describe spectroscopic observations of 2MASS $0532+8246$ obtained with the Near-IR Spectrometer (NIRSPEC: McLean et al. 1998, 2000) and Low Resolution Imaging Spectrograph (LRIS; Oke et al. 1995) at Keck Observatory. In $\S 4$ we derive the space motion of this object from the imaging and spectroscopic data. In $\S 5$ we discuss the substellarity of 2MASS $0532+8246$ based on subsolar metallicity models from Burrows et al. (2001). We discuss our results in $\S 6$.

\section{IDENTIFICATION OF 2 MASS $0532+8246$}

2MASS $0532+8246$ was identified in a search for $\mathrm{T}$ dwarfs using the 2MASS working database (Burgasser et al. 2002). It was selected for its blue NIR colors $\left(J-K_{s}=\right.$ $0.26 \pm 0.16)$ and lack of an optical counterpart at the

\footnotetext{
${ }^{10}$ Source designations for the 2MASS Point Source Catalog are given as "2MASS Jhhmmss[.]ss \pm ddmmss[.]s." The suffix conforms to IAU nomenclature convention and is the sexagesimal R.A. and decl. at the J2000.0 equinox. We adopt a shorthand notation of "2MASS hhmm \pm ddhh" throughout the remainder of this paper.
}

TABLE 1

Observational Properties of the Late-type sdL 2MASS $0532+8246$

\begin{tabular}{|c|c|}
\hline Parameter & Value \\
\hline$\alpha_{\mathrm{J} 2000.0}{ }^{\mathrm{a}}$ & $5^{\mathrm{h}} 32^{\mathrm{m}} 53^{\mathrm{s}} .46$ \\
\hline$\delta_{\mathrm{J} 2000.0^{\mathrm{a}}}$. & $+82^{\circ} 46^{\prime} 46^{\prime \prime} .5$ \\
\hline 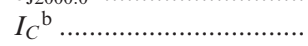 & 19.2 \\
\hline 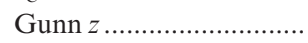 & $18.30 \pm 0.16$ \\
\hline 2MASS $J \ldots \ldots \ldots \ldots$ & $15.18 \pm 0.06$ \\
\hline 2MASS $H \ldots \ldots \ldots \ldots \ldots \ldots$ & $14.90 \pm 0.10$ \\
\hline $2 \operatorname{MASS} K_{s} \ldots \ldots \ldots \ldots \ldots \ldots \ldots \ldots \ldots \ldots$ & $14.92 \pm 0.15$ \\
\hline & $2 " .60 \pm 0.15 \mathrm{yr}^{-1}$ \\
\hline$\theta$ & $130^{\circ} .0 \pm 1.8$ \\
\hline$v_{\text {rad }}$ & $-195 \pm 11 \mathrm{~km} \mathrm{~s}^{-}$ \\
\hline
\end{tabular}

2MASS position in both POSS-I and POSS-II photographic plates. A finder chart is given in Figure 1, and astrometric and photometric data from 2MASS are listed in Table 1. Follow-up NIR imaging using the Palomar 60 inch Infrared Camera (IRCAM; Murphy et al. 1995) on 1999 September 25 (UT) determined that this object is not an uncataloged minor planet. Gunn $r$-and $z$-band images obtained with the Palomar 60 inch Facility CCD Camera on 2002 November 18 and 2003 January 25 (UT) further confirmed the red opti$\mathrm{cal} /$ NIR colors of this object. Aperture photometry from 2002 November 18, calibrated with observations of the SDSS photometric standard BD $+33^{\circ} 4737$ [Smith et al. 2002; correcting $\left(r^{\prime}, z^{\prime}\right) \rightarrow($ Gunn $r$, Gunn $z)$ using photometry from Kent 1985] yield $r>20.9$ (5 $\sigma$ upper limit) and $z=18.30 \pm 0.16$. As the NIR and optical/NIR colors of this object are consistent with those of other identified latetype L and T dwarfs (Kirkpatrick et al. 1999; Fan et al. 2000; Dahn et al. 2002), 2MASS 0532+8246 was initially believed to be a firm $\mathrm{T}$ dwarf candidate.

\section{SPECTROSCOPIC OBSERVATIONS}

\subsection{NIR Spectrum}

We obtained NIR spectra of 2MASS $0532+8246$ on 2002 December 24 (UT) using NIRSPEC on the Keck II $10 \mathrm{~m}$ Telescope. Conditions were clear with seeing of 0".6. We observed the object in three spectral configurations, N3
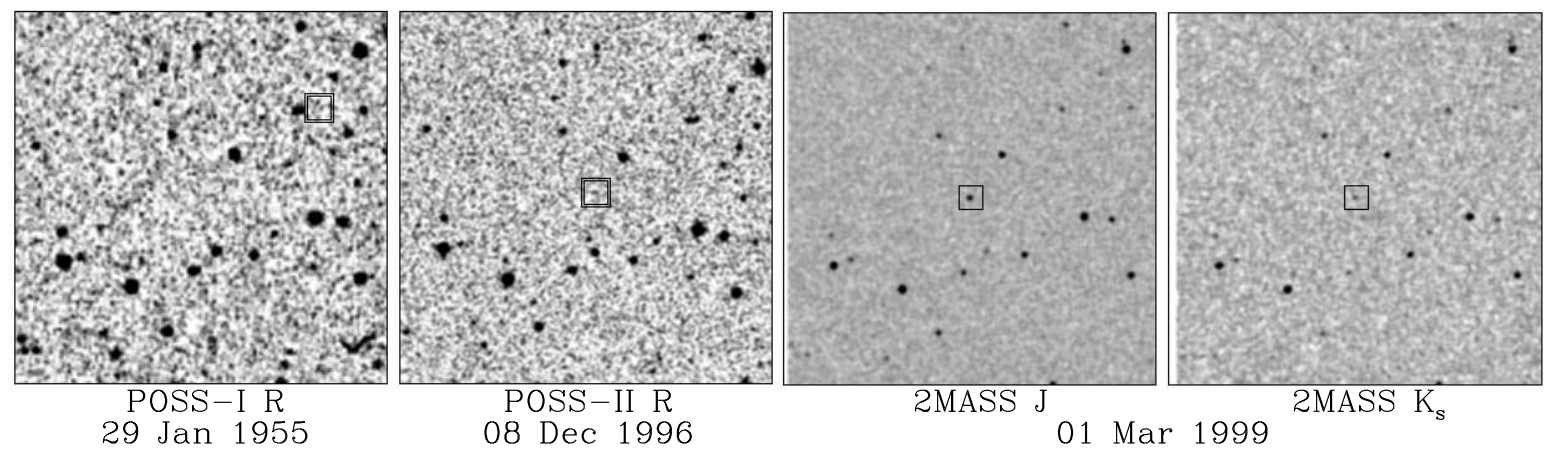

FIG. 1. - Finder chart for 2MASS $0532+8246$, showing POSS-I ( $R$ band), POSS-II ( $R$ band), and 2MASS ( $J$ and $K_{s}$ bands) fields. Images are scaled to the same spatial resolution, $5^{\prime}$ on a side, with north up and east to the left. A $10^{\prime \prime}$ box is centered at the position of the subdwarf in the 2 MASS images and at the extrapolated position (assuming $\mu=2$ ". 6 and $\theta=130^{\circ}$ ) in the POSS-I and POSS-II images. 
$(1.14-1.38 \mu \mathrm{m}), \mathrm{N} 5(1.51-1.79 \mu \mathrm{m})$, and N6b (1.94-2.32 $\mu \mathrm{m})$, corresponding roughly to the $J, H$, and $K$ NIR bands. Spectral resolution was $R \approx 2000$ for our 0 "!38 slit. For each observation, two exposures of $300 \mathrm{~s}$ each were obtained, dithering $20^{\prime \prime}$ along the slit. All observations were made in the air-mass range 2.6-3.0. Immediately after each sequence of exposures, internal flat-field and NeAr arc lamps were observed for calibration, and the A0 star HD 34360 was observed just before or after the target source in each spectral order at similar air masses. Data were reduced using the REDSPEC package, ${ }^{11}$ a detailed description of our reduction procedures is given in McLean et al. (2003). Final flux calibration of the individual spectral orders was done using 2MASS photometry; however, because the orders do not span the entire photometric bandpasses (Cutri et al. 2003), ${ }^{12}$ our final flux calibration may be somewhat underestimated at the $J$ band but reasonably correct for the $H$ and $K$ bands.

The resulting NIR spectrum is shown in Figure $2 a$. The $1.5-2.4 \mu \mathrm{m}$ spectral energy distribution is fairly smooth and blue, reminiscent of an early-type main-sequence star or white dwarf, and quite unlike the much redder NIR spectra of late-type $\mathrm{M}$ and L dwarfs (cf. overlay of the L7 DENIS 0205-1159AB in Fig. 2a; Delfosse et al. 1997; Kirkpatrick et al. 1999; McLean et al. 2003). However, the red optical/ NIR colors and optical molecular bands (see below) of 2MASS $0532+8246$ are inconsistent with a hot stellar atmosphere. We instead attribute the shape of the NIR spectrum to enhanced absorption by the CIA $\mathrm{H}_{2}$ 1-0 quadrupole band centered near $2.5 \mu \mathrm{m}$ (Saumon et al. 1994; Borysow, Jørgensen, \& Zheng 1997). This pressure-sensitive band, present in late-type $\mathrm{L}$ and $\mathrm{T}$ dwarfs (Tokunaga \& Kobayashi 1999; Burgasser et al. 2002) and cool subdwarfs (Leggett et al. 2000), is generally broad and featureless, consistent with the observed spectrum. Strong $\mathrm{H}_{2}$ absorption may explain the absence of $\mathrm{CH}_{4}$ absorption bands at 1.6 and $2.2 \mu \mathrm{m}$, the defining features of $\mathrm{T}$ dwarfs, and the 2.3 $\mu \mathrm{m} \mathrm{CO}$ band, a key spectral feature in $\mathrm{M}, \mathrm{L}$, and early-type $\mathrm{T}$ dwarf spectra. On the other hand, $\mathrm{H}_{2} \mathrm{O}$ absorption appears to be present at $1.3-1.5$ and $1.7-2.0 \mu \mathrm{m}$, and minor features throughout the $H$ band are likely attributable to weaker $\mathrm{H}_{2} \mathrm{O}$ lines. Two weak features at 1.573 and $1.626 \mu \mathrm{m}$ are present, possibly attributable to $\mathrm{FeH}$ absorption (Cushing et al. 2003) or poor correction of telluric $\mathrm{OH}$ lines; these features are not coincident with the $1.6 \mu \mathrm{m} \mathrm{CH}_{4}$ band. Overall, this portion of the spectrum of 2MASS $0532+8246$ is quite unlike any known late-type $\mathrm{M}, \mathrm{L}$, or T dwarf, based on its blue slope and absence of $\mathrm{CH}_{4}$ and $\mathrm{CO}$ bands.

The $J$-band spectrum, shown in detail in Figure $2 b$, is far more rich, with atomic lines of $\mathrm{K}_{\mathrm{I}}(1.1690,1.1773,1.2543$, and $1.2522 \mu \mathrm{m})$ and $\mathrm{Fe}_{\mathrm{I}}(1.1528,1.1886,1.1976,1.2128$, and $1.3210 \mu \mathrm{m})$, and strong molecular bands of $\mathrm{FeH}(1.194$ and $1.239 \mu \mathrm{m})$ and $\mathrm{H}_{2} \mathrm{O}(1.3 \mu \mathrm{m})$ observed. Above the spectrum we plot an opacity spectrum of $\mathrm{FeH}$ (Dulick et al. 2003), which shows that much of the fine structure observed is caused by this molecule. There are no lines of $\mathrm{Mg} \mathrm{I}, \mathrm{Ca} \mathrm{I}$, or $\mathrm{Al}$ I present in this spectral region, and the $\phi \mathrm{TiO}$ system around $1.25 \mu \mathrm{m}$ (Jørgensen 1994) is either absent or obscured by $\mathrm{FeH}$ absorption. The overall spectral mor-

\footnotetext{
${ }^{11}$ Additional information is available at

http://www2.keck.hawaii.edu/inst/nirspec/redspec/index.html.

12 Additional information on Cutri et al. (2003) is available at http://www.ipac.caltech.edu/2mass/releases/allsky/doc/explsup.html.
}

phology at $J$ band is quite similar to the L7 DENIS 0205-1159AB, with the notable exception of stronger $\mathrm{FeH}$ and $\mathrm{H}_{2} \mathrm{O}$ absorption.

\subsection{Optical Spectrum}

We obtained a red optical spectrum of 2MASS $0532+8246$ on 2003 January 3 (UT) using LRIS on the Keck I 10 m telescope. Conditions were clear with subarcsecond seeing. Two exposures of $1200 \mathrm{~s}$ each were obtained through the red channel, dithered by $2^{\prime \prime}$ between exposures along the $1^{\prime \prime}$ (4.7 pixels) slit. We employed the 400 line $\mathrm{mm}^{-1}$ grating blazed at $8500 \AA$, yielding $6300-10100 \AA$ spectra with $7 \AA$ resolution $(R \sim 1200)$. Dispersion on the chip was $1.9 \AA^{\circ}$ pixel $^{-1}$. The OG570 order-blocking filter was used to suppress higher order light. Observations of the B1 V flux standard Hiltner 600 (Hamuy et al. 1994) were obtained for flux calibration, and the G0 V star HD 38847 was observed after the target at similar air mass (2.4) for telluric calibration. HeNeAr arc lamp exposures were taken immediately after the target observations for wavelength calibration, and quartz lamp flat-field exposures (reflected off of the interior dome) were observed at the start of the night to correct for detector response. Data reduction procedures using standard IRAF ${ }^{13}$ routines are discussed in detail in Burgasser et al. (2003a).

The final flux-calibrated and telluric-corrected optical spectrum for 2MASS $0532+8246$ is included in Figure $2 a$ and shown in detail in Figure $2 c$. Strong metal hydride bands of $\mathrm{FeH}(8692,9896 \mathrm{~A}), \mathrm{CrH}(8611,9969 \mathrm{~A})$, and $\mathrm{CaH}$ $(6750 \AA)$ are clearly discernible. The $9896 \AA$ FeH WingFord band is the strongest seen in any cool spectrum to date, and absorption from the shorter wavelength $\mathrm{FeH}$ and $\mathrm{CrH}$ bands extends to roughly $9100 \AA$. Weaker bands of TiO $(7053,8432 \AA)$ and $\mathrm{H}_{2} \mathrm{O}(9250,9400 \AA)$ are also seen. We point out an absorption feature between 9570 and $9700 \AA$, which is too strong to be attributable to intrinsic $\mathrm{H}_{2} \mathrm{O}$ absorption, based on the weakness of the $9250 \AA$ band; it is also not properly placed for $\mathrm{FeH}, \mathrm{CrH}, \mathrm{TiH}$, or $\mathrm{CH}_{4}$ absorption. This band is weakly present in late-type $\mathrm{M}$ and early-type L dwarf spectra and may be a hitherto unrecognized metal hydride band. It is also possible, although unlikely, that this feature is attributable to residual telluric absorption, as telluric corrections were made only to $9650 \AA$ (the extent of the strong terrestrial $\mathrm{H}_{2} \mathrm{O}$ bands; Stevenson 1994). Individual alkali lines of $\mathrm{Na}$ I $(8183,8195 \AA), \mathrm{Rb}$ I (7800, $7948 \AA$ ), and Cs I (8521, $8943 \AA$ ) are present, while the resonance $\mathrm{K}$ I doublet $(7665,7699 \AA)$ is exceptionally strong and pressure-broadened, as seen in late-type $\mathrm{L}$ and $\mathrm{T}$ dwarfs (Kirkpatrick et al. 1999; Liebert et al. 2000). The red optical/NIR colors of 2MASS $0532+8246$ are clearly a consequence of the $\mathrm{K}$ I red wing; integrating the spectrum over the $I_{C}$ bandpass (Bessell 1990) yields $I_{C} \approx 19.2$ and $I_{C}-J \approx 4.0$, similar to mid- and late-type L dwarfs (Dahn et al. 2002). The blue end of the spectrum appears to be similarly suppressed by the pressure-broadened $\mathrm{Na}$ I doublet $(5890,5896 \AA)$. Both Li I ( $6708 \AA)$ absorption and $\mathrm{H} \alpha(6563$ $\AA$ ) emission lines are absent. The overall spectral morphology, excluding hydride bands and $\mathrm{TiO}$, is again quite similar

\footnotetext{
${ }^{13}$ The Image Reduction and Analysis Facility (IRAF) is distributed by the National Optical Astronomy Observatory, which is operated by the Association of Universities for Research in Astronomy, Inc., under cooperative agreement with the National Science Foundation.
} 

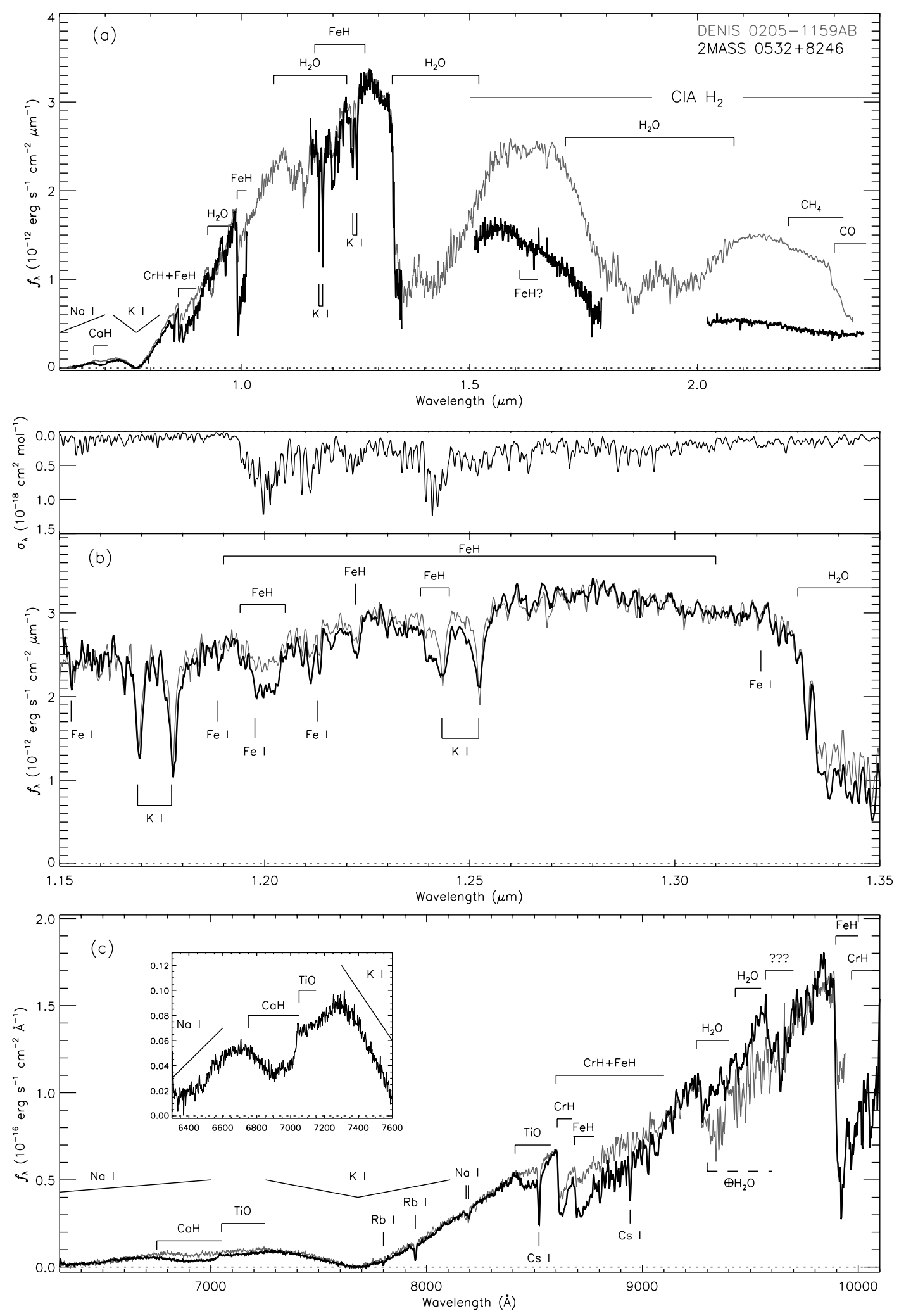

FIG. 2.-Spectrum of 2MASS $0532+8246$ (thick black line) as compared with the L7 DENIS 0205-1159AB (thin gray line: data from Kirkpatrick et al. 1999; McLean et al. 2003) In all panels, spectral data for 2MASS $0532+8246$ have been shifted by $v_{\text {rad }}=+195 \mathrm{~km} \mathrm{~s}^{-1}$, and data for DENIS 0205-1159AB have been scaled to coincide at $1.27 \mu \mathrm{m}$. Zero points are indicated by dotted lines. (a) Observed $0.63-2.35 \mu \mathrm{m}$ spectrum, with NIRSPEC bands scaled to 2MASS photometry. Key atomic and molecular features are indicated; note that the $2.2 \mu \mathrm{m} \mathrm{CH}_{4}$ and $2.3 \mu \mathrm{m}$ CO bands present in the spectrum of DENIS 0205-1159AB (McLean et al. 2001) are not present in that of 2MASS 0532+8246. (b) Top: FeH absorption coefficient vs. wavelength, from Dulick et al. (2003). Bottom: J-band spectrum of 2MASS 0532+8246, with line identifications for K I, Fe I, FeH, and $\mathrm{H}_{2} \mathrm{O}$. (c) Red optical spectrum, with key features indicated. Uncorrected telluric $\mathrm{H}_{2} \mathrm{O}$ absorption in the DENIS $0205-1159 \mathrm{AB}$ data is indicated by the dashed bracket. Inset window shows a close-up of the 6350-7600 $\AA$ spectral region, highlighting strong $\mathrm{CaH}$ and weak $\mathrm{TiO}$ bands; no $\mathrm{Li}$ I or $\mathrm{H} \alpha$ lines are seen. [See the electronic edition of the Journal for a color version of this figure.] 
to the L7 DENIS 0205-1159AB, suggesting a rather cool atmosphere.

\subsection{Characterization and Spectral Classification}

2MASS $0532+8246$ is not a $\mathrm{T}$ dwarf, based on the absence of the 1.6 and $2.2 \mu \mathrm{m} \mathrm{CH}_{4}$ bands (Burgasser et al. 2002; Geballe et al. 2002) and the presence of strong $\mathrm{FeH}$ and $\mathrm{CrH}$ and weak $\mathrm{TiO}$ and $\mathrm{Na}$ I features in the optical (Burgasser et al. 2003a). It appears that this object is instead a metal-deficient, late-type $\mathrm{L}$ dwarf. Enhanced CIA $\mathrm{H}_{2}$ and strong hydride bands in the optical and NIR spectra of 2MASS $0532+8246$ are consistent with a metal-poor atmosphere. Both are consequences of the overall reduced metal opacity, resulting in larger column abundances of the remaining chemical species and increased relative abundance of metal hydrides over double-metal species. The presence of the 7053 and $8432 \AA \mathrm{TiO}$ bands is somewhat at odds with this interpretation, particularly since $\mathrm{TiO}$ is generally weak or absent in disk dwarfs later than L5 (Kirkpatrick et al. 1999). One possibility is that the mechanism of Ti depletion in these objects-incorporation into multiple-metal condensates of $\mathrm{CaTiO}_{3}, \mathrm{Ca}_{3} \mathrm{Ti}_{2} \mathrm{O}_{7}$, $\mathrm{Ca}_{4} \mathrm{Ti}_{3} \mathrm{O}_{10}$, and $\mathrm{Ti}_{2} \mathrm{O}_{3}$ (Lodders 2002) - may be inhibited in the low-metallicity atmosphere of 2MASS $0532+8246$. Chemical equilibrium calculations incorporating subsolar abundances are required to address this hypothesis. The TiO bands may also indicate a somewhat warmer atmosphere than the L7 comparison object DENIS 0205-1159AB.

Classifying 2MASS $0532+8246$ is therefore not straightforward, particularly as there are no other metal-deficient L dwarfs currently known for direct comparison. The M subdwarf and extreme subdwarf sequences of Gizis (1997) terminate at sdM7 and esdM5.5, respectively, although recent late-type discoveries are classified via extrapolation and limited modifications of this scheme (Lepine, Rich, \& Shara 2003; Lepine, Shara, \& Rich 2003). Metal classification (d, sd, and esd) in the Gizis (1997) scheme is based on the strength of the $7053 \AA \mathrm{TiO}$ band, which is exceedingly weak in the spectrum of 2MASS $0523+8246$. Numerical classification is based on the $6750 \AA \mathrm{CaH}$ band, which is suppressed in this spectrum by $\mathrm{Na} \mathrm{I}$ and $\mathrm{K}$ I absorption. Thus, a simple extrapolation of the Gizis (1997) scheme fails in the $\mathrm{L}$ dwarf regime. While there is general agreement between the spectral morphologies of 2MASS $0532+8246$ and DENIS 0205-1159AB, the optical/ $J$-band hydride features and TiO bands are more similar to mid-type L dwarfs (Kirkpatrick et al. 1999; McLean et al. 2003). Additionally, there are no cool dwarf standards that match the 1.3-2.4 $\mu \mathrm{m}$ spectral region. We therefore tentatively classify this object a late-type sdL. Identification of additional metal-poor L subdwarfs may eventually allow the definition of a robust classification scheme.

\section{SPACE MOTION}

Examination of the IRCAM and CCD follow-up images show that 2MASS $0532+8246$ has moved significantly since it was first imaged by 2MASS. We therefore measured its proper motion by deriving its coordinates on each of the images using the 2MASS coordinates of background stars for astrometric calibration. Results are listed in Table 2. A linear fit yields $\mu=2$ ". $60 \pm 0$ ". $15 \mathrm{yr}^{-1}$ at position angle $130^{\circ} 0 \pm 1.8$, making 2 MASS $0532+8246$ one of the highest proper-motion stars known (Bakos, Sahu, \& Németh 2002). This object is not present in the LHS because of its optical faintness, although a faint counterpart may be present in the POSS-II R-band image at the extrapolated position (Fig. 1). Residuals in the proper-motion fit are indicative of parallactic motion, but positional uncertainties are too large for a reliable measure.

The radial velocity of 2 MASS $0532+8246$ was derived by cross-correlating its $J$-band spectrum with those of five early- and mid-type L dwarfs (chosen for their strong NIR $\mathrm{FeH}$ absorption) with measured radial velocities: 2 MASS 0746+2000AB, 2MASS 1439+1929, G196-3B, Kelu 1, and DENIS 1228-1547AB (Basri et al. 2000; Reid et al. 2002). All five of these objects have been observed as part of the NIRSPEC Brown Dwarf Spectroscopic Survey (McLean et al. 2003) and are therefore wavelength-calibrated in the same manner as the data presented here. We derive a heliocentric $v_{\text {rad }}=-195 \pm 11 \mathrm{~km} \mathrm{~s}^{-1}$, where the uncertainty is derived from the scatter of values among the comparison objects. This value is consistent with heliocentric velocity offsets in the $1.1690 / 1.1773 \mu \mathrm{m} \mathrm{K}$ I lines $\left(\left\langle v_{\mathrm{rad}}\right\rangle=\right.$ $-175 \pm 17 \mathrm{~km} \mathrm{~s}^{-1}$ ) and the optical Cs I and $\mathrm{Rb}$ I lines $\left(\left\langle v_{\mathrm{rad}}\right\rangle=-192 \pm 24 \mathrm{~km} \mathrm{~s}^{-1}\right)$.

The substantial radial velocity and proper motion of 2MASS $0532+8246$ identifies it as a high-velocity source. Determining its total space motion, however, requires an estimate of its distance. If we assume 2MASS $0532+8246$ is bound to the Galaxy (Galactocentric $v_{\text {tot }} \lesssim 500 \mathrm{~km} \mathrm{~s}^{-1}$; Carney, Latham, \& Laird 1988), we derive an upper limit distance of $54 \mathrm{pc}$ assuming $(U, V \text {, and } W)_{\odot}=$ $\left(10.00,5.25\right.$, and 7.17) $\mathrm{km} \mathrm{s}^{-1}$ (Dehnen \& Binney 1998) and local standard of rest (LSR) velocity $V_{\mathrm{LSR}}=220 \mathrm{~km}$ $\mathrm{s}^{-1}$ (Kerr \& Lynden-Bell 1986). A spectrophotometric parallax estimate is highly uncertain, given the substantial

TABLE 2

ASTROMETRY OF 2MASS $0532+8246$

\begin{tabular}{|c|c|c|c|c|c|}
\hline $\begin{array}{c}\text { Epoch } \\
\text { (UT) } \\
(1)\end{array}$ & $\begin{array}{l}\text { Instrument } \\
\text { (2) }\end{array}$ & $\begin{array}{c}\alpha_{\mathrm{J} 2000.0} \\
\text { (3) }\end{array}$ & $\begin{array}{c}\delta_{\mathrm{J} 2000.0} \\
(4)\end{array}$ & $\begin{array}{c}\sigma_{\alpha}{ }^{\mathrm{a}} \\
(\operatorname{arcsec}) \\
(5)\end{array}$ & $\begin{array}{c}\sigma_{\delta}^{\mathrm{a}} \\
(\operatorname{arcsec}) \\
(6)\end{array}$ \\
\hline $1999 \operatorname{Mar} 1$ (02:20). & 2MASS & 053253.46 & +824646.53 & 0.30 & 0.30 \\
\hline 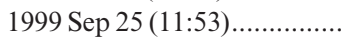 & P60 IRCAM & 053254.14 & +824645.60 & 0.07 & 0.06 \\
\hline 2002 Nov $18(08: 01) \ldots \ldots \ldots \ldots . .$. & P60 CCD & 053257.37 & +824640.33 & 0.35 & 0.23 \\
\hline 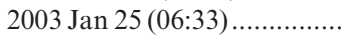 & P60 CCD & 053257.66 & +824640.00 & 0.37 & 0.22 \\
\hline
\end{tabular}

NotE.-Units of right ascension are hours, minutes, and seconds, and units of declination are degrees, arcminutes, and arcseconds.

a Uncertainty in $(\alpha, \delta)$ derived from standard deviation of positions for background stars. 
redistribution of flux in the NIR and uncertainty in the numeric subtype for this object. However, assuming that the $M_{I_{\mathrm{C}}}$ magnitude of 2MASS $0532+8246$ is similar to that of mid- to late-type L dwarfs (Dahn et al. 2002, $17 \lesssim M_{I_{\mathrm{C}}} \lesssim 19$ ), we derive a conservative estimate of $10-30$ pc. The corresponding heliocentric space velocities at the median distance are $(U, V$, and $W)=(-13,-301$, and 28) $\mathrm{km} \mathrm{s}^{-1}$. The substantial $V$ velocity, between -390 and -212 $\mathrm{km} \mathrm{s}^{-1}$ over the adopted distance range, indicates zero or retrograde motion with respect to the LSR and lends additional support that this object is a member of the halo population.

\section{IS 2 MASS $0532+8246$ SUBSTELLAR?}

Disk L dwarfs with spectral types later than $\sim$ L4 are predominately substellar (Gizis et al. 2000); hence, the late-type spectral morphology of 2MASS $0532+8246$ suggests that it too may be a brown dwarf. To test this hypothesis, we compared the estimated $T_{\text {eff }}$ of this object with subsolar metallicity evolutionary models from Burrows et al. (2001). In Figure 3, we plot $T_{\text {eff }}$ versus mass for ages 10 and $15 \mathrm{Gyr}$ and metallicities $Z=0.1,0.3$, and $0.01 Z_{\odot}$. At these late ages, the transition between stellar and substellar masses spans a substantial range in temperature and is more dramatic for lower metallicities due to the reduced atmospheric opacities and hence enhanced luminosities (Burrows et al. 1993).

Deriving an accurate $T_{\text {eff }}$ for 2MASS $0532+8246$ is difficult without adequate distance or bolometric flux measurements. Solar-metallicity mid- to late-type L dwarfs have temperatures $1400 \lesssim T_{\text {eff }} \lesssim 1800 \mathrm{~K}$ (Kirkpatrick et al. 1999;

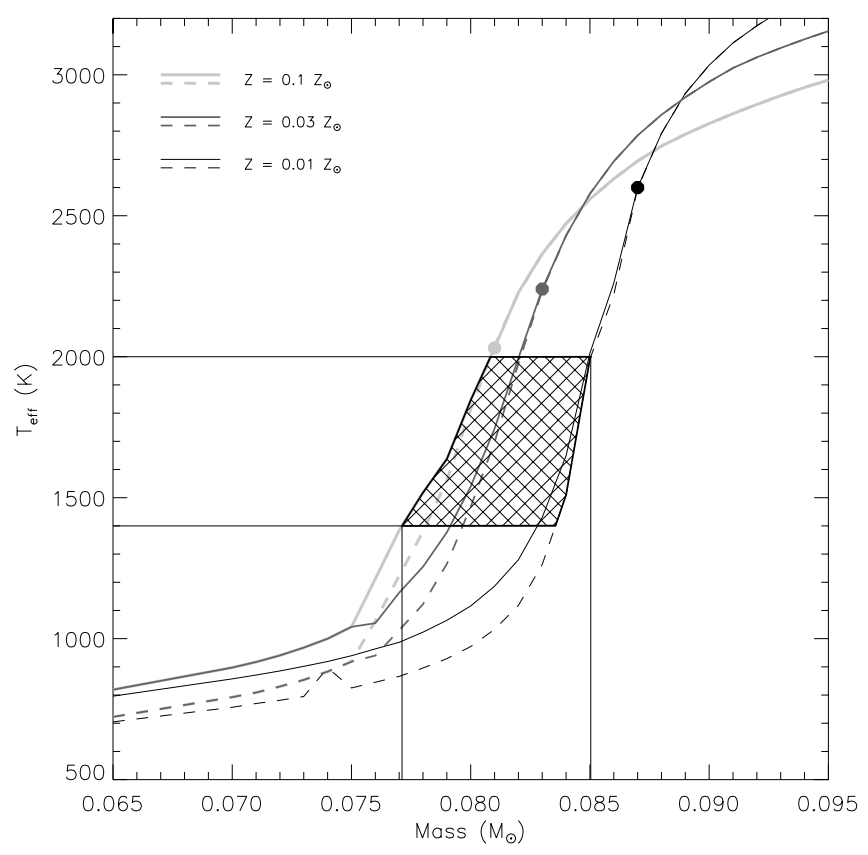

FIG. 3.- Shows $T_{\text {eff }}$ vs. mass for theoretical evolutionary models with $Z=0.1$ (light gray line), 0.03 (dark gray line), and 0.01 (black line) $Z_{\odot}$, and ages 10 (solid line) and 15 (dashed line) Gyr. The HBMMs at $10 \mathrm{Gyr}$ for all three metallicities are indicated by solid circles. Our adopted $1400 \lesssim T_{\text {eff }} \lesssim 2000 \mathrm{~K}$ range for 2 MASS $0532+8246$ corresponds to masses of $0.077 \leqslant M \leqslant 0.085 M$. over all values of metallicity and age shown (hatched region). For each metallicity the derived mass is below the corresponding HBMM.
Basri et al. 2000; Pavlenko, Zapatero Osorio, \& Rebolo 2000; Leggett et al. 2001; Stephens et al. 2001; Burgasser et al. 2002; Dahn et al. 2002). In general there is little difference between the temperatures of M-type subdwarfs and dwarf stars of the same numeric type (Gizis 1997; Leggett et al. 2000), although late-type M subdwarfs (e.g., the sdM7 LHS 377) may be 100-300 K hotter (Leggett et al. 2000). We therefore adopt a conservative temperature range of $1400 \lesssim T_{\text {eff }} \lesssim 2000 \mathrm{~K}$. We further assume that the metallicity of 2 MASS $0532+8246$ lies in the range $0.01 \lesssim Z \lesssim 0.1 Z_{\odot}$, typical for sdM and esdM stars (Gizis 1997; Gizis \& Reid 1997). For these parameters, we derive a mass range of $0.077 \lesssim M \lesssim 0.085 M_{\odot}$. Larger masses correspond to lower metallicities so that in each case the derived mass is below the HBMM. Therefore, we conclude that the 2MASS $0532+8246$ is probably substellar, although more refined estimates of its atmospheric properties through further observations and spectral modeling are required to validate this result.

\section{DISCUSSION}

The existence of a substellar subdwarf is not unexpected, as mass functions for the nearby halo population (Gizis \& Reid 1999) and globular clusters (Piotto \& Zoccali 1999) are shallow but rising, $d N / d M \propto M^{-(0.5-1.3)}$ at $M \sim 0.15 M_{\odot}$, similar to the disk population (Reid et al. 1999; Chabrier 2002). However, the steep drop in $T_{\text {eff }}$ and hence luminosity across the metal-poor substellar boundary implies that the vast majority of these halo brown dwarfs will have $T_{\text {eff }} \lesssim 1300 \mathrm{~K}$, i.e., in the $\mathrm{T}$ dwarf regime and cooler (Burgasser et al. 2002). Indeed, one possible T-type subdwarf has already been identified, 2MASS 0937+2931 (Burgasser et al. 2002), an object that also exhibits unusually strong CIA $\mathrm{H}_{2}$ and $\mathrm{FeH}$ absorption (Burgasser et al. 2003b) and a substantial proper motion (Burgasser et al. 2003b). Relatively few L-type subdwarfs should exist, however, as they span a much narrower range in mass at ages later than 10 Gyr. The discovery of 2MASS $0532+8246$ may therefore be quite fortuitous.

Given its optical faintness, we expect that few counterparts to this late-type L subdwarf will be identified in current proper-motion surveys. NIR proper-motion surveys, on the other hand, could potentially reveal more of these objects by detecting them at the peak of their spectral energy distribution. A sizeable fraction of the sky $(\sim 30 \%)$ has been scanned more than once by 2MASS during its 3 years of observations, and analysis of these data may identify new, very cool high-motion stars. A second-generation 2MASS survey could greatly improve on this work, adding substantially to the short list of very cool subdwarfs currently known.

A. J. B. acknowledges useful discussions with K. Cruz and $\mathrm{J}$. Wei-Chun during the preparation of the manuscript and thanks our anonymous referee for her/his helpful criticism. We are grateful for the assistance of our Keck Observing Assistants Joel Aycock and Gary Puniwai and Instrument Specialist Paola Amico, and our Palomar Telescope Operators, Karl Dunscombe and Barrett "Skip" Staples. We also acknowledge the 2MASS staff for their laudable efforts on the 2MASS database, without which none of this research could have been possible. A. J. B. acknowledges support by NASA through Hubble 
Fellowship grant HST-HF-01137.01, awarded by the Space Telescope Science Institute, which is operated by the Association of Universities for Research in Astronomy, Inc., for NASA, under contract NAS 5-26555. A. S. B. acknowledges funding through NASA grants NAG5-10760 and NAG5-10629. Portions of the data presented herein were obtained at the W. M. Keck Observatory, which is operated as a scientific partnership among the California Institute of Technology, the University of California, and the National Aeronautics and Space Administration. The Observatory was made possible by the generous financial support of the W. M. Keck Foundation. This publication makes use of data from the Two Micron All Sky Survey, which is a joint project of the University of Massachusetts and the Infrared Processing and Analysis Center, funded by the National Aeronautics and Space Administration and the National Science Foundation. 2MASS data were obtained through the NASA/IPAC Infrared Science Archive, which is operated by the Jet Propulsion Laboratory, California Institute of Technology, under contract with the National Aeronautics and Space Administration. POSS-I and POSS-II images were obtained from the DSS image server maintained by the Canadian Astronomy Data Centre, which is operated by the Herzberg Institute of Astrophysics, National Research Council of Canada. The authors wish to extend special thanks to those of Hawaiian ancestry on whose sacred mountain we are privileged to be guests.

\section{REFERENCES}

Bakos, G. A . Sahu, K. C \& Németh, P. 2002, ApJS, 141, 187

Basri, G., Mohanty, S., Allard, F., Hauschildt, P. H., Delfosse, X., Martín, E. L., Forveille, T., \& Goldman, B. 2000, ApJ, 538, 363

Bessell, M. S. 1990, PASP, 102, 1181

Borysow, A., Jørgensen, U. G., \& Zheng, C. 1997, A\&A, 324, 185

Burgasser, A. J., Kirkpatrick, J. D., \& Liebert, J., \& Burrows, A. 2003a, ApJ, in press

Burgasser, A. J., Kirkpatrick, J. D., McElwain, M. W., Cutri, R. M., Burgasser, A. J., \& Skrutskie, M. F. 2003b, AJ, 125, 850

Burgasser, A. J., et al. 2002, ApJ, 564, 421

Burrows, A., Hubbard, W. B., Lunine, J. I., \& Liebert, J. 2001, Rev. Mod. Phys., 73, 719

Burrows, A., Hubbard, W. B., Saumon, D., \& Lunine, J. I. 1993, ApJ, 406, 158

Carney, B. Latham, D., \& Laird, J. B. 1988, AJ, 96, 560

Chabrier, G. 2002, ApJ, 567, 304

Chabrier, G., \& Baraffe, I. 1997, A\&A, 327, 1039

Chamberlin, J. W., \& Aller, L. H. 1951, ApJ, 114, 52

Cushing, M. C., Rayner, J. T., Davis, S. P., \& Vacca, W. D. 2003, ApJ, 582, 1066

Dahn, C. C., et al. 2002, AJ, 124, 1170

Dehnen, W., \& Binney, J. J. 1998, MNRAS, 298, 387

Delfosse, X., et al. 1997, A\&A, 327, L25

Dulick, M., Bauschlicher, C. W., Jr., Burrows, A., Sharp, C. M., Ram, R. S., \& Bernath, P. 2003, ApJ, submitted (astro-ph/0305162)

Epchtein, N., et al. 1997, Messenger, 87, 27

Fan, X., et al. 2000, AJ, 119, 928

Geballe, T. R., et al. 2002, ApJ, 564, 466

Gizis, J. E. 1997, AJ, 113, 806

Gizis, J. E., Monet, D. G., Reid, I. N., Kirkpatrick, J. D., Liebert, J., \&

Williams, R. 2000, AJ, 120, 1085

Gizis, J. E., \& Reid, I. N. 1997, PASP, 109, 849

. 1999, AJ, 117, 508

Hamuy, M., Suntzeff, N. B., Heathcote, S. R., Walker, A. R., Gigoux, P., \& Phillips, M. M. 1994, PASP, 106, 566

Jørgensen, U. G. 1994, A\&A, 284, 179

Kent, S. M. 1985, PASP, 97, 165

Kerr, F. J., \& Lynden-Bell, D. 1986, MNRAS, 221, 1023

Kirkpatrick, J. D., et al. 1999, ApJ, 519, 802

Kuiper, G. P. 1939, ApJ, 89, 548

Leggett, S. K., Allard, F., Dahn, C., Hauschildt, P. H., Kerr, T. H., \& Rayner, J. 2000, ApJ, 535, 965

Leggett, S. K., Allard, F., Geballe, T., Hauschildt, P. H., \& Schweitzer, A. 2001, ApJ, 548, 908

Lepine, S., Rich, R. M., \& Shara, M. M. 2003, AJ, 125, 1598

Lepine, S., Shara, M. M., \& Rich, R. M. 2002, AJ, 124, 1190
Lepine, S., Shara, M. M. \& Rich, R. M. 2003, ApJ, 585, L69

Liebert, J., \& Probst, R. G. 1987, ARA\&A, 25, 473

Liebert, J., Reid, I. N., Burrows, A., Burgasser, A. J., Kirkpatrick, J. D., \& Gizis, J. E. 2000, ApJ, 533, L155

Lodders, K. 2002, ApJ, 577, 974

Luyten, W. J. 1979a, LHS Catalogue: A Catalogue of Stars with Proper Motions Exceeding 0".5 Annually (Minneapolis: Univ. Minnesota Press)

1979b, New Luyten Catalogue of Stars with Proper Motions Larger than Two Tenths of an Arcsecond (Minneapolis: Univ. Minnesota Press) (NLTT)

Martín, E. L., Delfosee, X., Basri, G., Goldman, B., Forveille, T., \& Zapatero Osorio, M. R. 1999, AJ, 118, 2466

McLean, I. S., Graham, J. R., Becklin, E. E., Figer, D. F., Larkin, J. E., Levenson, N. A., \& Teplitz, H. I. 2000, Proc. SPIE, 4008, 1048

McLean, I. S., McGovern, M. R., Burgasser, A. J., Kirkpatrick, J. D., Prato, L., \& Kim, S. 2003, ApJ, submitted

McLean, I. S., Prato, L., Kim, S. S., Wilcox, M. K., Kirkpatrick, J. D., \& Burgasser, A. J. 2001, ApJ, 561, L115

McLean, I. S., et al. 1998, Proc. SPIE, 3354, 566

Mould, J. R., \& Hyland, A. R. 1976, ApJ, 208, 399

Murphy, D. C., Persson, S. E., Pahre, M. A., Sivaramakrishnan, A., \& Djorgovski, S. G. 1995, PASP, 107, 1234

Oke, J. B., et al. 1995, PASP, 107, 375

Pavlenko, Ya.,Zapatero Osorio, M. R , \& Rebolo, R. 2000, A\&A, 355, 245

Piotto, G., \& Zoccali, M. 1999, A\&A, 345, 485

Reid, I. N., \& Hawley, S. L. 2000, New Light on Dark Stars (Chichester: Praxis)

Reid, I. N., Kirkpatrick, J. D., Liebert, J., Gizis, J. E., Dahn, C. C., \& Monet, D. G. 2002, AJ, 124, 519

Reid, I. N., et al. 1999, ApJ, 521, 613

Sandage, A. R., \& Eggen, O. J. 1959, MNRAS, 119, 278

Saumon, D., Bergeron, P., Lunine, J. I., Hubbard, W. B., \& Burrows, A. 1994, ApJ, 424, 333

Scholz, R.-D., Irwin, M., Ibata, R., Jahreiss, H., \& Malkov, O. Yu. 2000, A\&A, 353, 958

Schweitzer, A., Scholz, R.-D., Stauffer, J., Irwin, M., \& McCaughren, M. J. 1999, A\&A, 350, L62

Skrutskie, M. F., et al. 1997, in The Impact of Large-Scale Near-IR Sky Surveys, ed. F. Garzon (Dordrecht: Kluwer), 25

Smith, J. A., et al. 2002, AJ, 123, 2121

Stephens, D. C., Marley, M. S., Noll, K. S., \& Chanover, N. 2001, ApJ, 556, L97

Stevenson, C. C. 1994, MNRAS, 267, 904

Tokunaga, A. T., \& Kobayashi, N. 1999, AJ, 117, 1010

York, D. G., et al. 2000, AJ, 120, 1579 